\title{
How to Suceed in Convincing Municipalities to build Astronomy Centres: the experience of Campinas Region
}

\author{
By A.L.K. Bretones ${ }^{1} \&$ P.S. Bretones ${ }^{2}$ \\ ${ }^{1}$ Centro Educacional - SESI 403, Campinas, Brasil \\ ${ }^{2}$ Faculdade de Educação, Universidade Estadual de Campinas, Campinas, Brasil
}

\section{Introduction}

The objective of this work is to make known the astronomical activities in the region of Campinas, the process of developing municipal cooperation and the general conclusions that reflect this process.

This research has been done by means of interviews with people related to the creation of astronomical centers in the region of the city of Campinas that is located in the state of São Paulo in Brasil (Fig. 1 and 2).

The conditions studied are related with this region but many ideas could be used in developing countries or others.

Nowadays there are works in the areas of research, teaching and popularization in many institutions besides the individual efforts of teachers and amateur astronomers in general. At two local universities (Unicamp and Puccamp) the discipline of astronomy at the graduate level is still optional. There are no groups or departments of astronomy but various students have obtained the MSc degree in areas such as black holes, active galactic nuclei, cosmology and teaching of astronomy. There is also a small planetarium, a naked eye observatory, some astronomy clubs as well as various amateur astronomers who contribute with systematic observations of the sky in many fields such as Sun, planets, occultations, comets, astrophotography, CCD astronomy and even with the use of spectroheliograph, coronograph and a Schmidt camera. Some teachers conduct astronomical activities in many schools of the region within disciplines like sciences or physics as well as lectures, exhibits and observations of the sky. There are six municipal observatories (Campinas, Americana, Itapira, Piracicaba, Diadema and Amparo), with continuing activities of observational programs, public observation, exhibits, and other activities directed to students. These are not observatories that put an emphasis on research. They spend more time on educational, cultural and touristic activities.

With such a quantity of resources and assets we can ask: how can we succeed in convincing municipalities to build astronomy centers?

\section{The Process}

Various factors contribute to this. The first factor is the local conditions that helped this process. The city of Campinas has about one million people and a population of high cultural and economic level. Besides the existence of the universities, there is the proximity of São Paulo city, the great financial and population center of Brazil and also the existence of neighbouring cities of great agricultural, industrial and commercial production.

The observatory of Campinas was the first municipal observatory in the country and has influenced the opening of others. There have been astronomical activities in the city 


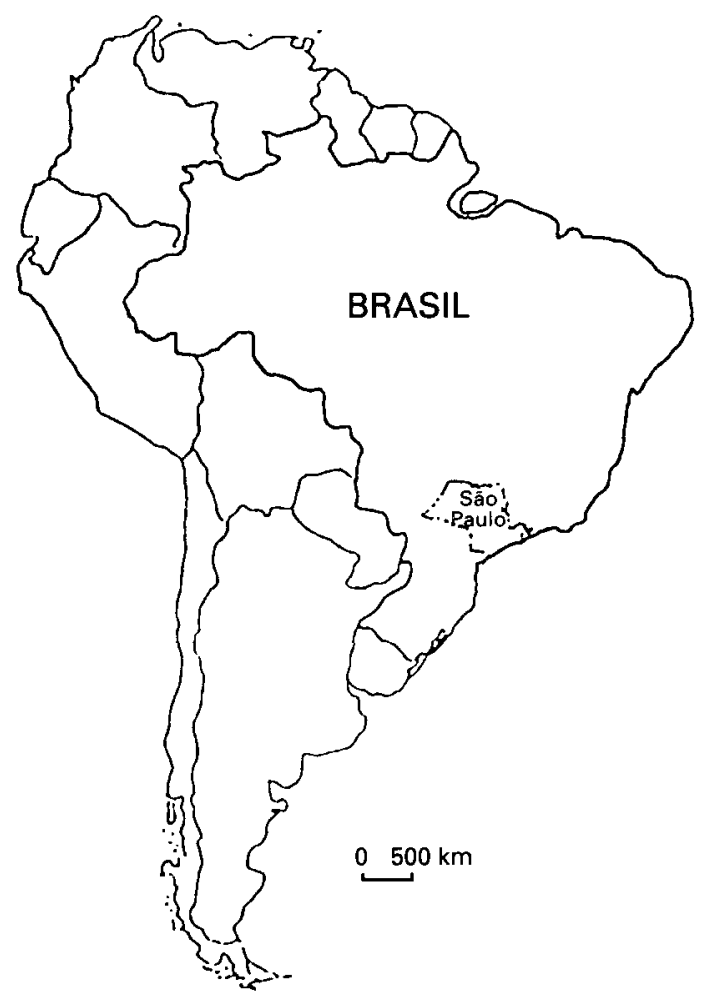

Figure 1. Geographical positions of Brazil in South America and the state of São Paulo in Brasil

of Campinas since the beginning of the century. There was an amateur astronomy group and teachers that besides activities in the university, lectured on astronomy.

In 1976 the mayor of Campinas, being also an amateur astronomer, looked to the Capricórnio Observatory staff to build a municipal observatory. That institution was established in another city and had already an academic background and high level amateurs. The mayor then summoned these people together with other astronomy teachers to staff the new observatory. The founders, J. Nicolini and N. Travnick and other teachers of the region had a great influence on the cultural development of the students and promoted it with their work and assistance of the media.

For a city to have an astronomy center it's necessary to be provided with a minimum of financial conditions and a population with good quality of life. It's even better if the city has some touristic potential and good geographical location. It's also important to have people interested in astronomy living in the city, an astronomy club or society where parallel events occur. Besides, it is important to have support from the media that increases the interest in astronomy matters and in reading of astronomy articles.

The last return of comet Halley influenced public opinion creating a strong lobby within the municipal administrations. Nowadays even without comet Halley, we have celestial phenomena that encourage these initiatives. Astronomy is always present in our lives. We can also take advantage of the passage of a comet, an eclipse, etc.

Its also true that people of good intellectual level, technical expertise and equipment are available for the city administration provided by the project's authors. The municipality does not spend much money, can use cheaper land in the outskirts, which is convenient for astronomical activities to build a small building. On the other hand, the municipality 


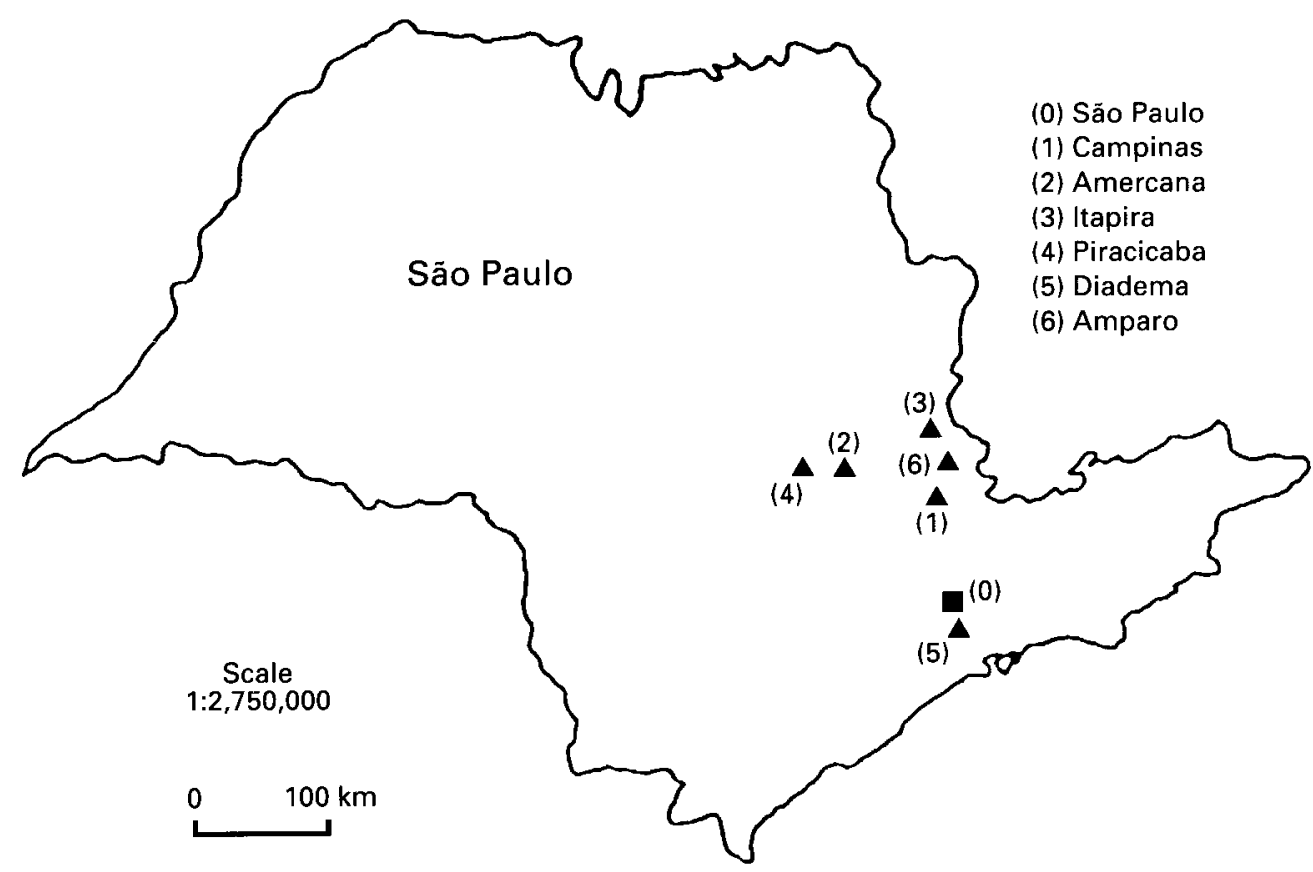

FIgURE 2. Geographical positions of the cities with minicipal astronomical centres: Campinas, Americana, Itapira, Piracicaba, Diadema and Amparo

also offers services to the community because it receives public and students in these centers, which is politically convenient. The municipality will therefore offer something accessible to the population and not merely an obsevatory dedicated just for research and a few interested people.

If the project's authors make available instruments, library, pedagogical material, and if possible, the dome, this also will be important to convince a municipality and show that it will not spend much money on the project.

It's important for the project's authors to know that it will be necessary to spend some of their own money in the beginning and in the maintenance of the undertaking.

This process takes time. First and foremost it is necessary to convince the secretary of culture or education that he must approve the project. Literature about the importance of the project must be well organized and as clear as possible. It is important to stress that news on astronomy happens on a daily basis, that people are interested and it has educational importance.

In general, the best occasion for a preliminary contact is after the first two years when the administration has paid the debts left by the former government and has taken care of other important projects. To initiate the preliminary contacts in the last year of administration is not advisable because it is carrying out projects already approved. But it is difficult to say which is the best occasion. It depends on many factors related to the administration's priorities. Another phenomenon typical of Brazil, is that there is some easiness for an astronomer to persuade the municipalities to build astronomy centers. This happens because we have a country where the government is formed by a learned elite that makes decisions without consulting the people - the vast majority. 
The most important aspect is to look for the right people for the first contact. This can be the secretary of culture or education and even one of the mayor's auxiliaries or someone who has influence over the decisions and can see the importance of an astronomy center. Many projects have not been established because the wrong persons were chosen for the first contacts. Sometimes its difficult to find the right person. One may have to try many times before someone happens to appear who is interested in the project.

The next step is to go to the mayor and to explain the idea and the political dividends. The project depends fundamentally on the mayor. If he does not like the idea the project will not be established. But this will not be enough; it's also important that the mayor's auxiliaries like the project. The administration's office staff, the cultural and educational people and the engineers should always be consulted. There have been cases when the mayor was accessable and wanted to carry out the project but it was blocked by his auxiliaries who alleged financial difficulties among others.

But when the mayor and his auxiliaries have will and vision of the importance, the process is faster. The city would not need to have an astronomy club or society or even previous support of the media. The mayor must issue a decree approving the project and later the city council should approve it. It is important to have the acceptance of the city councillors by political maneuvering without preference for any political party. If the community accepts the project it will be difficult for the councillors to block it for it now belongs to the city. It may be necessary to speak about the project in a city council session. It is necessary, that besides other important works, to establish that an observatory is also important. If we had only to think about the populations' problems there would not be education, culture and leisure.

Something that can make things easier is to take advantages of ongoing projects like an ecological park, a science museum etc. There you need to be flexible for the place must be of easily accessable to the public and visitors. Choosing a remote place, where the administration would have to install the basic infrastructure like running water, light, telephone, roads, etc, would make the project more expensive and make it more difficult to convince the municipality. Afterwards a contract of services and equipment lease are made. The contract is valid for a year and renewable. If the project's authors want to become employees of the municipality this would add additional problems. If the astronomical center is established by law, and there is community support, there will be employment stability.

As far as the planetarium of Campinas is concerned, it has been established as a result of the presence of the observatory. The federal government needed to install two telescopes and since the municipality decided to house them the city was presented with a planetarium. The federal government does not incur the risk of installing a planetarium in a city which does not have astronomical activities and potential to develop this field. The planetarium is a project accepted more easily by the municipality because it is profitable. Its sessions have a guaranteed public attendance since it does not have to rely on good weather.

If the observatory is situated in a big city and is having problems and if the municipality does not solve them, the commuinity and media demand the solution of such problems. But this is soon forgotten because the city has so many other problems to take care of and other attractions as well. The smaller the city the more attention an observatory gets because generally it is one of a few attractions in town. When there are problems they are taken care of by the appropriate channels without any further pressure from the media or the community. These problems are then internally solved. In a small city, people know the mayor, and demand the solution of several problems directly with him.

In the case of Brazil and other countries as well, these observatories can be used 


\section{THE PROCESS}

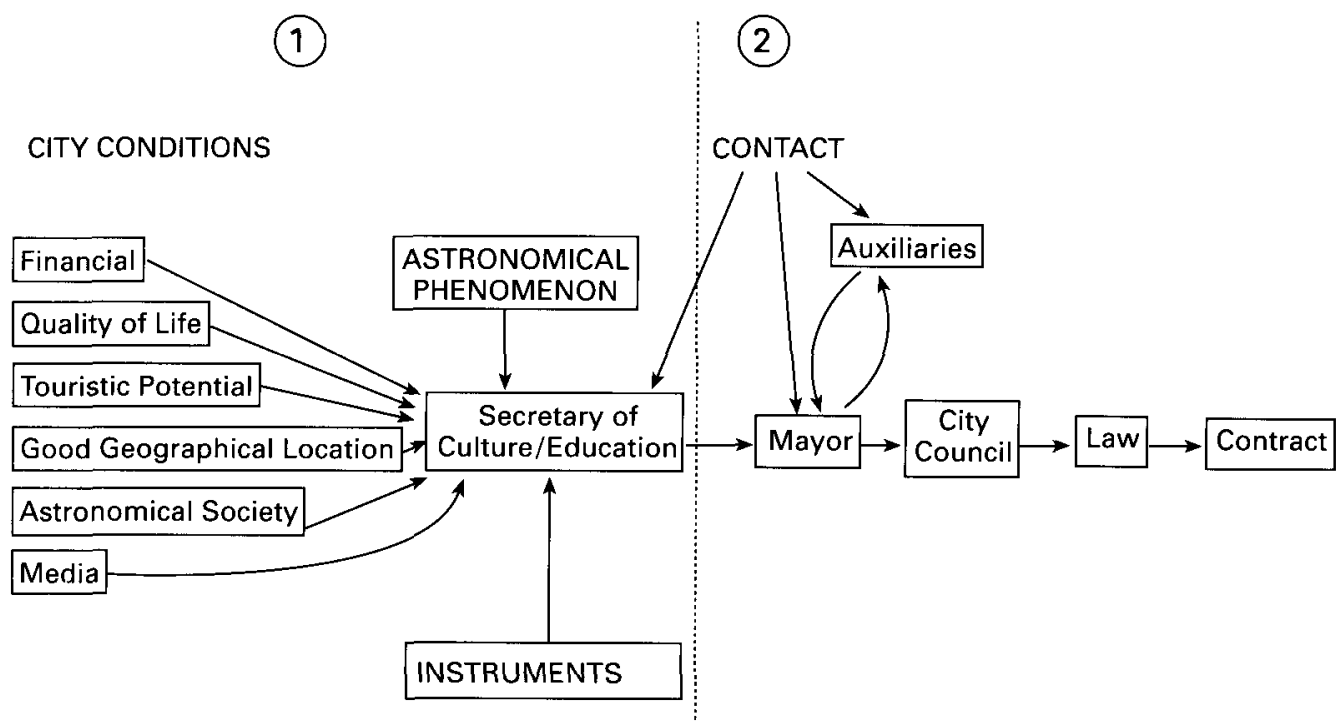

Figure 3.

as a means of employment of astronomers. As a municipal employee the astronomer does not have an adequate career plan. A municipality has no structure and means of assessment of the professional progression of an astronomer as it would in the university. The published papers, for instance, generally do not mean anything for the astronomer's career plan inside the municipality. In the case of an astronomer having a contract of services, his or her scientific production would be taken into account at the time of the contract's signature only. Later it would be of little value at the contract's renewal time. In a small city it much more difficult for the municipality's employees have low wages.

As a means of assessment the municipalities, in some cases, request an annual report. But the most important thing is to keep many activities frequently reported by the media. It must be well done work, so this way, the municipality recognizes that the astronomy center has been useful for the community. This way, the contracts are renewed every year. These projects can sometimes benefit from political opportunism. The mayor has a four year term and the observatory is usually built in the last two years and begins its operation in the final days of the term.

There have been instances where projects were cancelled after being approved. Furthermore, there have been cases where a municipal administration installed an observatory and the next administration abandoned the project. The establishment of an observatory in a city is a curious fact. Sometimes it has all the elements to be a successful project but it is not. Even after it has been approved by the city council the mayor may still have more interest in other works. By law the mayor has the power to veto such a project at any time and even against the community's interest. This would hardly happen in a region where an astronomy center has been established with the community's support.

Maintenance has been the great problem of these centers. It happens that people do 
not understand the importance of these projects. It is difficult to find two consecutive mayors who are well informed about the project. The fact that the astronomy center is established by law is no guarantee that it will thereafter function well.

After a mayor has established the astronomy center others usually continue to support the project but not so enthusiastically. They have not given all the necessary support but have not failed to recognize the importance of the project. In one case, the mayor almost extinguished the observatory, because of political rivalry with the former mayor. In smaller cities, with around 100,000 inhabitants, there are unique political characteristics. Once a political group is installed it is difficult to have any significant change in the status quo. In some cases this has been an advantage. A mayor may not support an established astronomy center but would not extinguish it because he is a friend of the former mayor. Every mayor gives more attention to their own projects as a way to have political gains. The problem is that the decisions can be imposed by a mayor who sometimes does not understand much about astronomy. He sometimes wishes to add inadequate or imcompatible improvements that can be deleterious to the project, e.g. street lights. It has been necessary to work this way because the mayors have only a political vision of the matter.

\section{Conclusions}

The process described has its problems because it can create instituitions directed by people without scientific background. There are no criteria for choosing the people who run the project. This process can be just political.

From our point of view we believe that the importance is not only to convince the municipalities but to persuade the people about the significance of these astronomical centers and by this way to influence the local government.

If the project is rooted in the people, children and parents, a new mentality can be created. This result has been better in smaller cities because its people are proud to have such an institution. In this case the people can be considered an ally and democracy is done.

An observatory should have more activities related to teaching in the schools. It would also be interesting to popularize astronomy in places out of the observatory such as city suburbs and even shopping centers or squares for it to be accessible for everyone.

\subsection{Acknowledgements}

We would like to express our deepest thanks to C. A. Argüello for the orientation of the project and A. C. Negreiros for the critical reading of the text and IAU for a Travel Grant. We wold like to thank Dr J. Percy for the suggestion of the subject of this research.

\section{References}

Special inquiry for this report.

Personal records of interviews with N. Travnick, C. Corsini and C. E. A. Mariano. Personal information from several institutions. 